\title{
A Review of Studies on Unconscious Emotional Priming
}

\author{
Juan Chen 1,2,3, He Chen1,2,3, Lingkai Ma1,2,3, Yuanyuan Jiang1,2,3 \\ ${ }^{1}$ Institute of Psychological Sciences, College of Education, Hangzhou Normal University, Hangzhou, China \\ ${ }^{2}$ Zhejiang Key Laboratory for Research in Assessment of Cognitive Impairments, Hangzhou, China \\ ${ }^{3}$ Center Cognition and Brain Disorder, Hangzhou Normal University, Hangzhou, China \\ Email: 592970734@qq.com
}

How to cite this paper: Chen, J., Chen, H., Ma, L.K. and Jiang, Y.Y. (2020) A Review of Studies on Unconscious Emotional Priming. Open Access Library Journal, 7: e6284. https://doi.org/10.4236/oalib.1106284

Received: March 31, 2020

Accepted: April 18, 2020

Published: April 21, 2020

Copyright $\odot 2020$ by author(s) and Open Access Library Inc.

This work is licensed under the Creative Commons Attribution International License (CC BY 4.0).

http://creativecommons.org/licenses/by/4.0/

\begin{abstract}
Emotional priming extends the priming paradigm to the field of emotion, which not only deepens the traditional priming paradigm, but also promotes the experimental study of emotion. There is a new development of automatic activation of unconscious emotions and attitudes, that is, the subjects did not know that the priming stimulus had been presented. His research is of great significance in promoting the research of attitude in the field of emotion and social cognition. As a new extension of the research paradigm of unconscious emotional priming, the paper mainly discusses the contribution of this research paradigm to the field of emotion and social cognition, the existing problems and the future research direction.
\end{abstract}

\section{Subject Areas}

Psychology, Unconsciousness

\section{Keywords}

Emotional Priming, Unconsciousness, Emotion

\section{Introduction}

Priming effect has been one of the main experimental paradigms in cognitive research. Recent studies have found that when priming and detecting stimuli have the same emotional color, the subjects' responses are different from those of the control group, which is called emotional priming. The existence of this phenomenon injects vitality into the study of emotion and finds an experimental paradigm for the study of emotion [1]. The study of emotional priming began in the early 1980s and involves both conscious and unconscious priming. Unconscious emotions start previously rendered below the threshold stimulation (usually with 
emotional words or images), or take a masking stimulus presentation styles, interval after a certain period of time, enter the test stage, is to try and start the stimulus of the same or related perform a task, or with the launch of a new stimulus has nothing to do perform this task as a baseline measurement of operation, old and new stimulus is adopted to improve the operating performance, as a result of difference constitutes the repeated start measuring [2]. At present, the emotional activation of implicit memory, implicit memory, the naming or judgment of people or things, and so on. Priming is also known as "subliminal priming" because priming occurs so quickly that it cannot be consciously perceived.

\section{Unconscious Emotional Priming Occurs}

\subsection{The Concept of Unconscious Priming}

One of the classic experiments on subliminal emotional priming: Murphy and Zajone's (1993) judgment preference experiment. Illiterate subjects were then presented with a picture of a positive or negative face for four milliseconds, followed by a Chinese character and asked to judge whether it was "good" or "bad". The results were as follows: the subjects were more likely to assign the meaning of "good" to the words of the happy emoticons, and the meaning of "bad" to the words of the angry emoticons. If the expression time of the picture was changed to 1000 milliseconds, the subjects consciously perceived the expression, and the definition of words was rarely affected by the facial expression. Similarly, at the behavioral level, Bargh and Pietromonaco asked participants to perform a task that required concentration. The subjects were presented with words related to the hostile nature of the personality, then asked to read a description of the person, and then asked to rate the person. The results showed that the more hostile words the subjects were presented with in advance, the more negative their evaluation of the target was. On the physiological level, when Ohman and Soars studied the unconscious anxiety of phobia patients, they presented a neutral picture of patients with ophiophobia and their normal faces, and measured their electrodermal responses [3]. Images of snakes, spiders, flowers and mushrooms were shown for 30 milliseconds before the images were rendered. The results showed that subjects with a fear of snakes had significantly lower skin resistance in the snake-led test [4].

A study by Winkielman, Berridge, and Wilbarger (2005) expands on the traditional concept of "unconscious emotions." In this study, Winkielman et al. proposed that the occurrence of such emotions is an unconscious process when emotions are detected indirectly by behavior or physiological measurement and individuals have no accompanying conscious emotional experience. They asked participants to rate whether they were hungry or hungry. They were then presented with a subliminal series of emotional emojis (happy, neutral and angry). Then, the subjects were asked to judge the gender of the neutral faces presented on the threshold. After completing the gender determination task, the subjects were randomly divided into two groups. One group rated their current emotional experience (mood and arousal), followed by beverage consumption or beverage 
price evaluation. The other group had the opposite order. The final results support Winkielma and Berridge (2004)'s earlier view that the emotional responses of thirsty subjects inferred from subliminal emotional stimuli and fruity beverage consumption behaviors are unconscious [5].

According to Fazio's pioneering research, emotional priming is an automatic processing process. First, he found that there was an emotional priming of the priming stimulus at $300 \mathrm{~ms}$ of SOA presentation time. Second, subliminal priming also produces emotional priming. At present, there is an experimental separation between suprarenal and subliminal emotional priming.

\subsection{Unconscious Emotion Primes Paradigm}

\subsubsection{Wiens Model}

The so-called "Wiens model", namely the information processing model of unconscious emotions proposed by Wiens and Ohman [6], indicates two meanings of unconscious emotions: 1) the subjects are not aware of the emotional inducers, for example, they do not know why they are "angry"; 2) the subjects were not aware of the occurrence of the induced emotions, such as whether they were "angry or not".

\subsubsection{Background Masking Paradigm}

Masking technology mainly includes three paradigms: background masking, sandwich masking and energy masking. They have the same principle as the target, among which background masking is the most widely used [6]. The background masking technique is emphasized here. As defined by Carlson and Reinke (2010), the stimulus initially presented in background masking technology (i.e., the priming stimulus) is quickly masked by the stimulus subsequently presented (i.e., the masking stimulus). "Masking stimuli" are thought to interfere with the processing of "primes" in the sensory cortex, thereby impeding or even replacing the representation of primes [7]. Hendler et al. (2003) proposed that background masking technique can effectively control the level of consciousness of emotional stimuli [8]. Pessoa (2005) it was pointed out that the threshold of individual consciousness was reached if the masking stimulus presented $30 \mathrm{~ms}$ [9]. Unconscious emotions may be induced when the presence of emotional priming is less than $30 \mathrm{~ms}$.

\subsubsection{Experimental Dissociation of Affective Priming}

Wong et al. (2003) pointed out that the sensitivity of threshold and subliminal emotional priming to the repeated presentation of stimuli is different, and the latter is more sensitive to repetitive stimuli, and the differences in priming effects caused by different emotional titer priming stimuli are also different in different types of emotional priming effects [10].

Musch proposed that in the supraliminal emotional priming, a higher proportion of consistent valences of initiation stimulus and target stimulus produced a more pronounced emotional priming than a lower proportion of consistent valences, while subliminal emotion priming did not have this phenomenon. Greenwald et al. showed that the inconsistent word-pair relationship of the previous 
trial reduced the level of emotion priming of the next trial, but not influence the degree of subliminal emotional priming [11]. In 1993, Barrs and McCovern, using a contrastive analysis technique, found that the effects of subliminal emotion priming even outweighed the effects of supraliminal emotional priming. Although these two priming belongs to semantic priming, but the behavioral and electrophysiological evidences had shown the experimental dissociation of consciousness and unconsciousness.

\section{The Factors Influence the Unconscious Emotion Priming}

\subsection{The Sandwich Masking of Priming Stimuli}

Zheng (2003) does not immediately evaluate the emotional intensity after presenting emotional or non-emotional stimuli, but asks the participants complete certain tasks first. If an emotional stimulus picture appears, participants should distinguish how much color does the picture contains in two minutes; if it is a non-emotional stimulus (triangle or ellipse), participants should determine which area is larger within two minutes. The results found that the masked picture produced a significant unconscious emotional priming effect [12].

In the Murphy and Zajone study, words or visual shape stimuli were used. Words were used as emotional masking stimuli, and shapes were used as non-emotional masking stimuli. Participants' emotional records were self-evaluated. It was found that the masked emotional words had a significant priming effect [13]. Ellis et al. stimulated participants' sadness and neutral emotions through masked words, and then asked the participants to evaluate whether the sentences were simple or complex. The assessment stage presented the sentences at a rate of 7 seconds or 10 seconds per sentence (Participants don't know if you want to recall the target word after evaluating the sentence). After evaluating the sentence, immediately present the sentence without the target word and ask to recall the target word. The results show that if sentences are presented at a speed of 7 seconds per sentence, there is a priming effect of sad emotions on the removal of the target words (in simple and complex sentences), and if the sentences are presented at a speed of 10 seconds, there is a priming effect of sad emotions on the removal of the target words (just in simple Sentence).This shows that the time effect of unconscious emotion priming is reflected in different tasks of diverse difficulties [14].

\subsection{Reaction Window Technology}

In the study of Klinger et al., subjects were required to perform indirect and direct tasks, and the results were analyzed by regression procedure. In the indirect task, the presentation time of the anterior masking stimulus, the prime word and the posterior masking stimulus were respectively $150 \mathrm{~ms}, 50 \mathrm{~ms}$ and $17 \mathrm{~ms}$, followed by the target stimulus of $333 \mathrm{~ms}$, and the response window of $133 \mathrm{~ms}$ after the target stimulus was presented, which was indicated by the black exclamation mark. After the reaction window time is exhausted, the black exclamation mark turns red and remains on the screen until the subject responds, and the subject is 
required to evaluate the emotional potency of the target stimulus as soon as possible before the exclamation mark turns red [15]. The intercept size in the regression program represents the magnitude of the priming effect at the unconscious level of the priming stimulus. Research shows that the intercept effect reaches a significant level, indicating the existence of subliminal emotional priming effect.

\subsection{Emotional Simon Paradigm}

In Houwer's (1998) research, nouns and adjectives with different emotional valences were asked to perform pronunciation tasks (naming or pronunciation of target stimuli) according to the grammatical classification of words. For example, the target stimulus belonging to nouns are named "positive" or "flower", while the target stimulus belonging to adjectives are named "negative" or "cancer". The study shows that the response time of matching relationship between stimulation titer and emotional valence (such as a bee is a positive titer) is shorter than non-matching relationship (e.g., a cockroach is a negative titer). In other words, the response time of valence nouns is shorter than that of negative valence nouns, and the response time of negative valence adjectives is shorter than that of positive valence adjectives [16]. Even though the experimenter explicitly instructed the subjects to ignore the emotional valence of the words, there was still a significant emotional activation phenomenon. Simon and Rudell (1967) reported the first Simon-type effect, participants are shown the left and right auditory words through the left and right speakers of the headset. They press a left or right key according to the meaning of the word, but ignore where it appears. The main finding is that participants cannot ignore the position of words: even though the positions of words change randomly, they cannot provide information about stimulus characteristics or responses, but if the position of words corresponds spatially to the response, the speed of the participants will be faster. See Figure 1 for details.

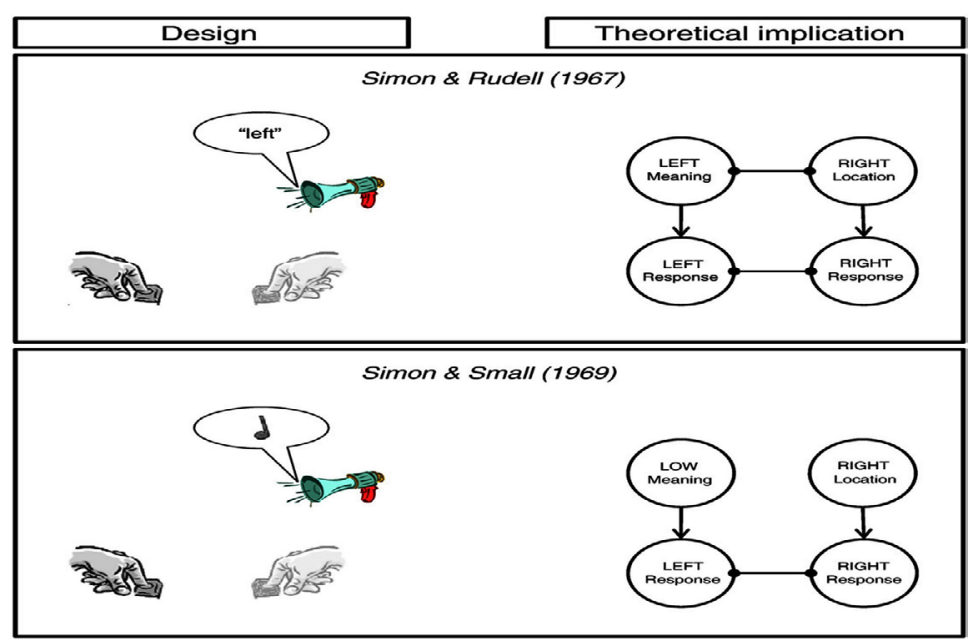

Figure 1. The designs of Simon and Rudell (1967) and Simon and Small (1969) and their theoretical implications. 


\section{The Effect of Unconscious Emotion Priming on Attention, Memory and Judgment}

\subsection{The Effect of Unconscious Emotion Priming on Attention}

Bruner (1994) [17] and Jennifer (1999) believe that information with similar emotional stimuli is more attractive to processors. When individuals pay more attention to a certain emotion change, it will be more noticeable to processors once this subtle emotion reappears in the later cognitive process.

Recent studies have shown that unconsciously fearful faces can be processed even under non-attentional conditions (Wang et al., 2012) [18]. Many studies have shown that: mammals (including humans) can unconsciously process emotional sensory stimuli (Panksepp, 2010) [19]. "Unconscious processing" of emotional stimuli can induce instinctive emotional behaviors in individuals (MacLean, 1990) [20]. Some researches on unconscious emotional priming on attention mainly focus on the unconscious level of individuals, while there are few researches on the level of consciousness or attention. Future researches may extend to the influence of unconscious emotional priming on attention.

\subsection{The Effect of Unconscious Emotion Priming on Memory}

\subsubsection{Breakthrough of Unconscious Emotion in Subliminal Emotional Priming}

Dietrich and Waller (2001) found that the subjects' negative and positive effects were significantly stronger than that of the neutral emotional words, 450 to 650 MS after receiving the stimulation when they study the influence of emotional components on the recognition behavior in the stimulation, that is, unconscious emotion has significant impact on semantic memory. Windmann and Kutas's (2001) research shows that emotional stimuli can cause "bias" in the process of cognitive memory, which means whether or not the stimulus object reappears, it responds "previously", the frontal cortex will relax the recognition threshold for negative emotional objects [21].

\subsubsection{Influence of Emotional Words and Cognitive Speed on Unconscious Emotional Initiation}

Challis and Krane's research on the cognition speed of words in different initiation states found that the emotional states that are expected to be initiated, whether positive or negative, are faster than those in the neutral state [22]. Moreover, the research of Wenture (2000) subliminal emotional relationship between target stimulus for the target stimulus processing and its mechanism, when start the stimulus in the experimental group and the emotional coloring of detecting target stimulus, while the control group in the start stimulating distractions for neutral stimulus, the experimental group to detect stimulation reaction time reaction than the control group significantly lengthened accordingly [23].

\subsubsection{Stem Complement or Sentence Complement Research on Unconscious Emotional Priming}

The subjects were shown a list of words, a word list was displayed with stems of 
English words such as "wor ...", and the first word appeared in their heads to complete the word. It was found that in the groups that induce unconscious negative emotions or anxiety, depression patients use word and Worry to complete the stemming, while unconscious positive emotion inducers use work, world, worm, etc. to complete more [24].

\subsection{Research on Judgment of Unconscious Emotion}

Ask participants to make emotional judgments about things (pictures or words) with emotional stimuli. Povell and Hemsley found that when words or pictures are presented to subjects at a rapid rate (below the threshold level), patients with negative emotions (such as anxiety) and depression can easily identify neutral words as negative words. This shows that unconscious negative emotions have a semantic priming effect on understanding part-of-speech, but Macleod and Mathews (1991) noticed in the research that the above phenomenon only occurs when people with depression or negative emotion inducers choose many targets. Only one the target does not occur as described above [25].

\section{Generating Mechanism and Main Models of Unconscious Emotion Activation}

\subsection{The Theory of the Mechanism of Unconscious Emotion Activation}

The association theory of emotion and memory occupies an important position in cognitive psychology. For a long time, both the initiation of unconscious emotion and the activation of conscious emotion have been carried out under its theoretical framework.

\subsubsection{Feeling Information Equivalence}

In the case of insufficient information or short time, people tend to use emotional feelings instead of cognitive judgment. When there is insufficient information about the judgment or the time is short, the complicated judgment is transformed into the subject's own emotional response to the goal. The emotional initiation that occurs is the subject's "misreading" of previous feelings, and when the source of such feelings is reasonably suspected, the information value of the feelings diminishes.

\subsubsection{Emotional Priority}

This theory indicates that in the human psychological structure, the emotional system that evaluates the meaning of the stimulus and the cognitive system that recognizes and understands the meaning of the stimulus are separate and interact with each other, but there are differences in the conditions and time for the effect. Emotional reactions require very few stimuli to appear and can occur in a very fast time; emotions are an independently developed and constructed psychological building block with its specific independent functions. 


\subsubsection{Automatic Diffusion Activation Theory}

The theory of automatic diffusion activation tends to treat emotions as memories of a particular kind of knowledge, and emotional reactions are the states of such memories when they are activated. All these processes can occur consciously or automatically. When a pattern or schema is automatically activated to take effect, it appears as a subliminal emotional activation effect.

\subsection{Other Models of Unconscious Emotion Activation}

Many participants did not observe the emotional priming effect at the conscious level. For this reason, some other models have been proposed for the influence of emotion on behavior initiation: "attention model" and "additive model".

\subsubsection{Attention Model}

This model is derived from the parallel reaction competition mechanism in the unconscious mechanism. Participants will respond to the item, when an individual respond to a response item, when the evidence of a certain response item exceeds a certain threshold. Mathew et al. (1998) tested subjects' attention bias that induced unconscious anxiety. The test material was threat words and stemming were performed. The results showed that both implicit and implicit subjects had attention bias towards threat words, but it is most significant at the implicit level, which proves that implicit memory is stronger than implicit memory in the emotional priming effect [26].

\subsubsection{Add Model}

Jennifer (1999) believes that certain unconsciousness can induce the emergence of specific emotional patterns in people, prompting people to produce a diffuse emotional state. This state is added to other states through unconsciousness, which initiates human behavior [27].

\section{Summary and Outlook}

At present, Chinese research on emotion activation is still at the stage of introducing foreign research examples, and there are few empirical studies on unconscious emotion activation. In general, there is a lack of in-depth discussions on the effects of emotional activation time in China, such as the relationship between emotional activation time, activation stimulus presentation time, and activation effect in different age groups. The relationship between the effect of subliminal emotional priming and decision-making, and the factors that affect the decision-making problems encountered in life, need to further explore the research of unconscious emotional priming.

The scope of unconscious emotion research is very wide, not only can be applied to the field of psychotherapy, such as creating a certain mood in psychoanalytic treatment can promote the recovery of this state-dependent memory. In addition, the unconscious emotional initiation has a greater effect on psychological treatment. For example, during the treatment process, patients with emo- 
tional disorders can be guided to understand negative or positive words of both sexes. If long-term training can make the emotionally disabled persons form an automatic reaction process, it can target training of patients during the treatment process to change the negative automatic response process to the information, thereby eliminating anxiety symptoms [28]. Research initiated by unconscious emotions also has a certain effect on personality. Domestic research on emotions and personality is just beginning. The transformation of emotions and the discussion of sound personality is a realistic issue. The study of the effects of emotional unconscious initiation may provide a theoretical basis for clinical psychologists. Generally speaking, the subliminal emotional priming effect opens a new path for the study of emotion and physiology, making the two more closely related.

In general, the unconscious emotional priming paradigm is of great significance to promote the study of emotions and attitudes in the field of social cognition. As a new research paradigm, the unconscious emotional priming paradigm opens up a new field for emotion research and provides new clues for studying unconscious perception. In addition, unconscious emotional priming also provides new directions for the development of emotions and brain science.

Through the introduction of unconscious emotional priming, this article systematically sorts out the generating mechanism and influencing factors of unconscious emotional priming, and its influence on attention, memory, and judgment. Our literature summary shows that the unconscious emotional priming paradigm can directly measure the impact of different emotional words on the subject's perception, attention, memory, etc., therefore, providing more intuitive evidence for the impact of subliminal emotions on people. However, there is still controversy about the generating mechanism and models of unconscious emotional priming, which may be a direction for future researches.

And this article has some limitations. Firstly, this article focused on the sources of the unconscious emotional priming paradigm and its impact on attention, memory, judgment, etc., but did not explore how unconscious emotional priming affects social decisions such as recruitment and fair distribution behavior. In addition, there is no sufficient evidence on how the unconscious emotional priming would affect the human brain mechanism, therefore, this article has not systematically sorted it out. This may be an exploration direction for future literature.

\section{Funding}

Juan Chen and He Chen were supported by the provincial advantage displine construction, talent cultivation, and research innovation from the College of Education, Hangzhou Normal University.

\section{Conflicts of Interest}

The authors declare no conflicts of interest regarding the publication of this paper. 


\section{References}

[1] Liao, S.L. and Tao, D.Q. (2004) New Progress in Research on Unconscious Emotion Priming. Psychological Science, 27, 701-704.

[2] Liu, H.C. and Xu, Y. (2009) The Influence of Subliminal Emotional Priming on Interpersonal Judgment. Psychological Research, 29.

[3] Bargh, J.A. and Pietromornaco, P. (1982) Automatic Information Processing and Social Perception: The Influence of Trait Information Presented Outside of Conscious Awareness on Impression Formation. Journal of Personality and Social Psychology, 43, 437-449. https://doi.org/10.1037/0022-3514.43.3.437

[4] Ohman, A. and Soares, J.J.F. (1994) Unconscious Anxiety: Phobic Response to Mask Stimuli. Journal of Abnormal Psychology, 103, 231-240.

https://doi.org/10.1037/0021-843X.103.2.231

[5] Winkielman, P. and Berridge, K.C. (2004) Unconscious Emotion. Current Directions in Psychological Science, 13, 120-123. https://doi.org/10.1111/j.0963-7214.2004.00288.x

[6] Wiens, S. and Öhman, A. (2002) Unawareness Is More than a Chance Event: Comment on Lovibond and Shanks. Journal of Experimental Psychology: Animal Behavior Processes, 28, 27-31. https://doi.org/10.1037/0097-7403.28.1.27

[7] Enns, J.T. and di Lollo, V. (2000) What's New in Visual Masking? Trends in Cognitive Sciences, 4, 345-352. https://doi.org/10.1016/S1364-6613(00)01520-5

[8] Hendler, T., Rotshtein, P., Yeshurun, Y., Weizmann, T., Kahn, I., Ben-Bashat, D. and Bleich, A. (2003) Sensing the Invisible: Differential Sensitivity of Visual Cortex and Amygdala to Traumatic Context. NeuroImage, 19, 587-600. https://doi.org/10.1016/S1053-8119(03)00141-1

[9] Pessoa, L., Japee, S. and Ungerleider, L.G. (2005) Visual Awareness and the Detection of Fearful Faces. Emotion, 5, 243-247. https://doi.org/10.1037/1528-3542.5.2.243

[10] Wong, P.S. and Root, J.C. (2003) Dynamic Variations in Affective Priming. Consciousness and Cognition, 12, 147-168. https://doi.org/10.1016/S1053-8100(03)00007-2

[11] Greenwald, A.G. and Klinger, M. (1995) Activation by Marginally Perceptible (“Subliminal”) Stimuli: Dissociation of Unconscious from Conscious Cognition. Journal of Experimental Psychology: General, 124, 22-24. https://doi.org/10.1037/0096-3445.124.1.22

[12] Zheng, X.F. (2003) An Experimental Study of Different Emotional Patterns Was Initiated by Visual Stimuli. Journal of Psychology, No. 3, 352-356.

[13] Murphy, S.T. and Zaione, R.B. (1993) Affect, Cognition and Awareness: Affective Priming with Sub Optimal and Optimal Stimulus. Journal of Personality and Social Psychology, 34, 123-139.

[14] Dosher, B.A. (1998) The Response-Window Regression Method-Some Problematic Assumptions: Comment on Draine and Greenwald. https://doi.org/10.1037/0096-3445.127.3.311

[15] Klinger, M.R., Burton, P.C. and Pitts, G.S. (2000) Mechanism of Unconscious Priming I. Response Competition, Not Spreading Activation. Journal of Experimental Psychology: Learning, Memory, and Cognition, 26, 441-455. https://doi.org/10.1037/0278-7393.26.2.441

[16] Houwer, J.D. and Eelen, P. (1998) An Affective Variant of the Simon Paradigm. Cognition and Emotion, 12, 45-61. https://doi.org/10.1080/026999398379772 
[17] Niedenthal, P.M., Kitayama, S. and Bruner, J.S. (1994) The View from the Heart's Eye Emotional Influences in Perception and Attention. Academic Press, San Diego, CA, 269-284. https://doi.org/10.1016/B978-0-12-410560-7.50018-1

[18] Wang, L.L., Fu, S.M., Feng, C.L., Luo, W.B., Zhu, X.R. and Luo, Y.J. (2012) The Neural Processing of Fearful Faces without Attention and Consciousness: An Event-Related Potential Study. Neuroscience Letters, 506, 317-321. https://doi.org/10.1016/j.neulet.2011.11.034

[19] Panksepp, J. (2010) Affective Consciousness in Animals: Perspectives on Dimensional and Primary Process Emotion Approaches. Proceedings of the Royal Society B: Biological Sciences, 277, 2905-2907. https://doi.org/10.1098/rspb.2010.1017

[20] MacLean, P.D. (1990) The Triune Brain in Evolution. Plenum Press, New York.

[21] Windmann, S. and Kutas, M. (2001) Electrophysiological Correlates of Emotion Induced Recognition Bias. Journal Cognitive Neuroscience, 13, 877-891. https://doi.org/10.1162/089892901750363172

[22] Mandal, M.K., Jain, A.H., Nizamie, S., et al. (1999) Generality and Specificity of Emotion-Recognition Deficit in Schizophrenic Patients with Positive and Negative Symptoms. Psychiatric Research, 87, 39-46. https://doi.org/10.1016/S0165-1781(99)00047-5

[23] Wentura, D. (2000) Dissociative Affective and Associative Printing Effects in the Lexical Decision Task: Yes versus No Responses to Word Targets Reveal Evaluative Judgment Tendencies. Journal of Experimental Psychology: Learning, Memory, and Cognition, 26, 456-496. https://doi.org/10.1037/0278-7393.26.2.456

[24] Challis, H. and Krane, R.B. (1993) Affect Cognition, and Awareness: Affective Priming with Sub Optimal and Optimal Stimulus. Journal of Personality and Social Psychology, 64, 723-739. https://doi.org/10.1037/0022-3514.64.5.723

[25] Macleod, C. and Mathews. A. (1991) Biased Cognitive Operations in Anxiety Accessibility of Information or Assignment of Processing Priorities. Behavior Research and Therapy, 29, 599-611. https://doi.org/10.1016/0005-7967(91)90009-R

[26] Mathews, A., Mogg, K., May, J.I. and Evseck, M. (1998) Implicit and Explicit and Explicit Memory Bias in Anxiety. Journal of Abnormal Psychology, 98, 236-240. https://doi.org/10.1037/0021-843X.98.3.236

[27] Jennifer, L.M. and Cynthia, E.Z. (1999) Intensifying the Dominant Response: Participant-Observer Differences and Nonconscious Effects. Communication Research, 18, 371-388.

[28] Liu, R.H. and Wang, L. (2000) Subliminal Emotional Priming. Psychological Science, 23, 352-365. 